\title{
Risks in Oncology and Radiation Therapy
}

\author{
Adriano Marcolongo, Glauco Cristofaro, \\ Aldo Mariotto, Maurizio Mascarin, \\ and Fabio Puglisi
}

\section{Learning Objectives}

This chapter aims to promote awareness by providing essential scientific elements about the risks associated with clinical oncology, with a particular focus on chemotherapy and radiotherapy.

Here as follows is the range of covered subjects:

- First, the epidemiologic and cultural context is addressed in order to understand the peculiarity of oncology, a medical discipline in which the risk is directly related to the daily

\footnotetext{
A. Marcolongo $(\bowtie) \cdot$ A. Mariotto

IRCCS Centro di Riferimento Oncologico, National

Cancer Institute, Aviano, PN, Italy

e-mail: aldo.mariotto@cro.it

G. Cristofaro

Ophthalmology, Hospital University Careggi,

Florence, Italy

M. Mascarin

AYA Oncology and Pediatric Radiotherapy Unit, IRCCS Centro di Riferimento Oncologico, National Cancer Institute, Aviano, PN, Italy

e-mail: mascarin@cro.it

F. Puglisi

Department of Medicine, School of Medical Oncology, University of Udine, Udine, Italy

Unit of Medical Oncology and Cancer Prevention, Department of Medical Oncology, IRCCS Centro di Riferimento Oncologico, National Cancer Institute, Aviano, PN, Italy e-mail: fabio.puglisi@uniud.it
}

clinical practice, almost inseparable from the intervention that must be conducted, probably more than in any other branch of medicine.

- To increase specific knowledge, in a public health perspective, described herein is the epidemiology of the different adverse effects, listed according to their frequency of occurrence. The central topic of modern health organization in relation to the existing volume of activities, herein limited to surgery, and the quality of health services, which can arouse debates of professional and organizational nature. Finally, safety practices, which were proven to be effective, and the strategies to implement them are also addressed.

\subsection{Introduction}

Developments in science and technology, together with the improved organization of health systems, have allowed remarkable progress in cancer diagnosis, care, and rehabilitation. In terms of assessment and management of the clinical risk, however, considerable issues are raised by the continuously increasing frequency of the disease, the super specialization in care, the elevated media attention, and the growing competence of patients and their associations.

Due to the complexity of their disease, cancer patients have access to different methodologies 
and therapeutic strategies, all of which can be used with both curative intent and palliation:

- Cancer surgery

- Medical oncology

- Chemotherapy

- Hormonal therapy

- Immunotherapy

- Drug targeting

- Gene therapy

- Cell therapy

- Radiation therapy

- External beam radiotherapy

- Brachytherapy

- IORT intraoperative radiotherapy

- Radiometabolic therapy

Several therapeutic strategies can be adopted for treating oncology patients, including specific or combined/adjuvant approaches. Each strategy carries risks and adverse effects, which are not always acceptable, with the aim to achieve real benefits with the undertaken treatment.

The different, possible causes of these adverse events or errors, which can be frequent in certain situations, are discussed in this chapter.

Additionally, the chapter focuses on several strategies and organizational options aimed to minimize the risk and reduce therapeutic side effects, which can be negligible in some instances but very severe in others.

\subsection{The Epidemiological Context}

Tissue damage is unfortunately unavoidable during radiation therapy and chemotherapy, as both approaches work by means of a mechanism of destruction of nucleic structures essential to cancer cells, in order to reduce cell multiplication. The setback is that most healthy cells are just as susceptible as cancer cells to the damaging effects of the treatments. Radiotherapy is based on ionizing radiations that must go through healthy tissues in order to reach the tumor. Even though new technologies are being developed, each more sophisticated and better able to precisely target the cancerous areas, the tissues sur- rounding the tumor still tend to get damaged, but this is also due to the fact that the tissues surrounding cancer cells are susceptible to microscopic invasion by the malignant cells. Chemotherapy, as a pharmacological, systemic treatment, exerts its effects on the whole organism. Therefore, although in theory therapy could eliminate cancer cells, in practice it is often impossible as it becomes difficult to expose all cancer cells to adequate doses without damaging healthy tissues. Hence, the central issue of clinical oncology consists in balancing cancer treatment effects and the effects that therapies could have on the healthy tissues, reaching an equilibrium that, for a particular patient with a specific cancer type, the best possible therapeutic ratio could be obtained, meaning the best possible balance between the risk of damaging healthy cells versus killing cancer cells.

It is therefore accepted by the scientific community that collateral damage to healthy cells in oncology is often unavoidable. In other words, it has been accepted that during treatment with curative intent, adverse effects, including severe ones, can be expected to occur more than in other medical fields. Adverse events can occur during and after treatments with radiotherapy and with chemotherapy. Short-term or acute adverse effects occur during or shortly after treatment, and they usually last for a few days or up to 2 weeks. Long-term or chronic adverse effects are more common in radiotherapy than in chemotherapy. They occur usually after treatment and sometimes even years later. These effects are usually irreversible and sometimes yield slowly progressive outcomes with severe consequences in terms of morbidity and, therefore, in terms of complaints and legal actions [1].

Approximately, one third of people may develop cancer during their lives. Today, about $40 \%$ of cancer cases are curable. However, a number of patients can keep the disease under control for long periods of time though not achieving a complete recovery. As can be expected, at least half of them will die from their disease. The reason the numbers of simple complaints and legal actions have dramatically increased in recent years lies in the high mortality 
of the disease and the high toxicity rates of therapies, on the one hand, and in the higher expectations of citizens regarding health services, on the other. This new perspective has been favored by higher cultural resources and by a higher awareness of their own rights due to democratic progress.

The main areas of risk in clinical oncology include misdiagnosis (e.g., anticancer treatment delivered to patients without cancer, or to patients who had cancer in the past without disease recurrence, or for the wrong type of cancer), medication errors in preparation or administration, therapeutic toxicity, and negligence [1].

Two more categories can be added: delayed treatments, due to a delayed diagnosis, or delayed initiation or continuation of therapy, causing harm to the patient; over diagnosis risks in oncology (e.g., PSA and TSH).

These are the most frequent categories of unfavorable outcomes afflicting patients. Some of the distinguishing characteristics of the specialty of oncology should be kept in mind. Oncology differs above all in the central role of the dedicated nurses and technicians who are specifically trained in the administration of sophisticated therapies. In radiotherapy in particular, a peculiar specificity exists, namely medical physicists who are called upon to run systematic quality checks of the linear accelerators' performance and to realize radiation treatment plans. Furthermore, oncology has come to act according to multidisciplinary and multiprofessional organizational models, following specific decisional protocols of clinical behavior.

\subsection{Epidemiology of Adverse Effects}

The most frequent adverse effects of cancer therapies belong to the category of toxicity. In general, radical treatments can result in very severe adverse effects in a small proportion of patients and in moderately severe adverse effects in a bigger proportion, even when conducted correctly and in accordance with to the appropriate clinical direction. The studies which have investigated the epidemiology of adverse effects mainly relate to chemotherapy.

In a cohort of 449 patients with cancer of the breast or lung or colon-rectum who underwent chemotherapy and followed up for a median time of 6 months, $86 \%$ reported at least one adverse effect during the study period and $27 \%$ an adverse effect of fourth degree, most often fatigue or dyspnea. Fatigue was the most frequent effect (85\%), followed by diarrhea (74\%), and constipation (74\%). Prevalence and incidence rates of the adverse effects were similar among all types of cancer, and older age represented the only significantly associated demographic factor [2].

In another cohort study, patients in treatment with antineoplastic drugs were followed up for 2 years. Overall, 591 cases of adverse effects were reported, a $58.6 \%$ incidence rate. The prevalence of adverse effects was recorded among women (constituting $73 \%$ of the cohort). Of the patients with adverse effects, $50.2 \%$ requested a treatment, $12.9 \%$ were deemed severe cases, $87 \%$ moderate, and $51 \%$ unpredictable [3].

An important further study was conducted on 458 oncologic patients followed up for 8 months, investigating hospital stay. Among the unplanned hospital admissions, $13 \%$ were caused by a pharmacological event, $13 \%$ by an adverse drug reaction, and $2 \%$ by a major interaction between different drugs. In conclusion, one in 10 unplanned hospital admissions of cancer patients was caused by a pharmacological effect [4].

It is estimated that between half and two thirds of new cancer cases receive treatment plans that include radiotherapy. Seventy-five percent of them are aimed to cure the patients. Radiotherapyrelated adverse effects are the most frequent and they are described below.

Prevalence of depression among cancer patients is extremely variable, ranging from $0 \%$ to $60 \%$ in different case studies, according to study criteria, methodology, and populations. Depression is associated in particular with cancers of the oro-pharynx, lung, breast, brain, and pancreas, but rarely with gynecologic tumors and colorectal cancers. Since comorbidity and treatment regimen are combined, in particular, with chemotherapy, it is often difficult to evaluate the 
direct effect of radiotherapy on depression as well as on several other symptoms.

Patients often indicate fatigue as the most disturbing adverse effect of radiotherapy, more than pain, nausea, and vomit. Fatigue correlated with radiotherapy occurs acutely in $80 \%$ of patients and chronically in $30 \%$ of patients. For this reason, patients should be evaluated for this symptom at regular intervals.

Dermatitis caused by radiation is a common adverse effect of radiotherapy, often complicating treatment of breast, prostate, perineum, and head and neck cancers. It is, however, difficult to evaluate the real burden of the phenomenon as clinical practice in this field is biased by unreliable and contradictory evidence. Early reactions include skin rash and dry and humid exfoliation, while delayed events include pigmentation changes, telangiectasias, hair loss, atrophy, and ulcerations.

Concerning vascular diseases due to radiotherapy, a well-known adverse effect occurs mostly in Hodgkin lymphoma patients, and in lower numbers, in breast and lung cancer patients. It is estimated that the relative risk of cardiovascular events after mediastinal radiation ranges from $2.2 \%$ to $7.2 \%$ for Hodgkin lymphoma cases and from $1 \%$ to $2.2 \%$ for cancer of the left breast.

Concerning lung effects, pneumonia due to radiation occurs in $5.15 \%$ of patients undergoing radiation for mediastinal cancers and breast and lung cancers. The risk is directly associated with the volume of the irradiated lung, intensity of radiation, and any concurrent chemotherapy. Toxicity of the digestive tract due to damage to the salivary glands is common in the radiation of the head and neck, especially in the case of concurrent chemotherapy. Esophagitis is also a common, early adverse effect whose frequency grows with the increasing of radioactive doses and in the presence of concurrent chemotherapy. Acute enteritis after radiotherapy is usually a selflimiting process by means of a correction to the diet and the administration of anti-diarrheal medications. Usually, the symptoms start 3 months after the end of radiotherapy and can last indefinitely. Another frequent symptom is the emesis induced by radiotherapy. The main risk factors include the completion of chemotherapy before radiation of the upper abdomen and the width of the irradiated areas. Patients who receive totalbody radiations are at higher risk. Acute bladder infection, including the most severe hemorrhagic cystitis, is a less common effect of radiotherapy, and the risk is augmented by concurrent chemotherapy.

Sexual dysfunctions, including impotence, are common after radiotherapy for cancers of the prostate and, to a lesser extent, of the colonrectum. They are primarily a concern for older patients, who show a higher frequency of prostate cancer diagnosis. Erection dysfunction is more common with brachytherapy and with external radiotherapy. Among women, sexual dysfunctions are more common after radiotherapy for cervical and endometrial cancers. The adverse effects include a lower sex drive, vaginal dryness, and general sexual dissatisfaction.

\subsection{Medication Errors in Oncology Practice}

The publication of the report "To err is human" by the Institute of Medicine of Washington (IOM) in 1999 has led to a radical change in healthcare organizations with regard to the understanding of the phenomenon of medical error. In oncology, the current definition of medication error, i.e., any "preventable event that may cause or lead to inappropriate medication use or patient harm while the medication is in the control of the healthcare professional, patient, or consumer," is provided by the National Coordinating Council for Medical Error Reporting and Prevention.

A recent review of the literature from 1980 to 2017 has shown that medical errors in chemotherapy occur at a frequency ranging from 1 to 4 cases per 1000 prescriptions, concerning at least $1-3 \%$ of patients and appearing in all phases of the cure process [5]. The definition of medication error applies to all areas of medicine but can have different implications depending on the complexity of the discipline and the magnitude of the potential damage it can cause. 
Cancer care is going through a revolutionary period both in the diagnostic and the therapeutic fields. At the same time, the substantial increase in scientific data is making the system increasingly complex and constitutes a challenge for health professionals [6]. For example, the current rate of new therapeutic indications in hematologyoncology is about one per week, preventing the general medical oncologist from keeping up.

In oncology, several factors may expose patients to increased risk that can result in serious adverse effects (AEs) [5]. The greater vulnerability of cancer patients may be due to the fragility induced by the disease itself, to the narrow therapeutic index of many anticancer agents, or to the use of innovative therapies of which potential side effects and their management are not fully known. In addition, cancer care is often provided by inter-professional teams that need to be perfectly in tune when it comes to communication [7].

Quite surprisingly, although oncologists pay high attention to treatment-related toxicities of anticancer agents, oncology as a discipline lags behind other areas of medicine in focusing on understanding the nature of medication errors and the extent of their effects. Of note, only a few studies have analyzed the incidence and consequences of medication errors in oncology [5, 8].

In December of 1994, a tragedy occurred that turned the spotlight on to the need to work systematically to ensure and strengthen safety measures in the administration of anticancer therapies. The incident occurred at the Dana-Farber Cancer Institute in Boston, a Harvard teaching hospital and a prestigious US institution, and caused the death of a young health journalist, Betsy A. Lehman, who died of a massive overdose of chemotherapy for breast cancer. A similar event occurred 2 days apart and caused permanent heart damage in a patient cared for by the same medical team. Both patients were treated with autologous stem-cell transplant, in the context of an experimental protocol that included high doses of cyclophosphamide. However, because of misinterpretation of the study protocol and subsequent miscalculated dosage, they received about four times the intended dose. The error was missed by other doctors, nurses, and pharmacists, including some senior members of the team.

Further attention was drawn to the importance of safe administration and management of chemotherapy after another lethal event occurred in December of 2015, two decades after the death of Betsy Leman. The death of a 49-year-old man caused again by an unintended chemotherapy overdose catalyzed a call to action for healthcare systems to recognize and to implement safety principles and practices to prevent patient harm. Increased awareness and sensitivity regarding the risk of errors related to anti-tumor treatments and, in particular, to chemotherapy, has led the American Society of Clinical Oncology (ASCO) and the Oncology Nursing Society (ONS) to set standards to minimize treatment-related risks. The most updated version of the standards was published in 2016 and focused on four main points [9]:

- The environment and routine procedures

- Treatment planning and patient education before the start of treatment

- Specific standards for ordering, preparing (including labeling), and administering treatment (chemotherapy)

- Monitoring adherence to, and toxicity from, chemotherapy to promote safety both while on treatment and subsequently

In particular, great attention is paid to the definition of the healthcare setting that includes the policy to ensure the relevant qualifications of the various professional figures involved in the order, preparation, and administration of the treatments.

Special emphasis is placed on training programs, on participation in basic life support courses by the clinical staff, and on information that must be reported in the medical record and verified before treatment (e.g., diagnosis, stage of illness, clinical history, physical examination, history of allergies, level of information shared with and understood by the patient and/or caregivers, description of the treatment plan). In addition, the healthcare setting includes a policy on documents that standardizes the process for 
obtaining treatment consent and valid proof of consent. Patients must be sufficiently informed about the treatment plan, the potential side effects, the management of adverse events, the handling of medicines at home, the follow-up visits, and the procedures they may be subjected to for monitoring during the therapy. Furthermore, patients must be provided with contact information in case of need.

Regarding therapeutic orders, it is recommended that they be signed by hand or electronically. Verbal orders are not allowed, with the exception of those for holding or stopping therapy. New orders or changes to orders (e.g., dose adjustments) must be documented in the medical record. It is important that a chemotherapy order be extremely detailed and include the following information: the patient's name, a second patient identifier, the date of the prescription order is written, the regimen or protocol identifier (name and number), the cycle number and day, all medications listed using full, generic names, the drug dose (to be written following the standards regarding abbreviations, trailing zeros, and leading zeros), the dose calculation, the date of administration, the route of administration, the presence of allergies, any supportive care treatments appropriate for the regimens (e.g., premedications, hydration, growth factors), parameters that would require holding or modifying the dose (e.g., laboratory tests, patient clinical status), the sequencing of drug administration, and the rate of drug administration. Special recommendations are given for prescriptions of oral chemotherapy.

The standards also include requirements for the preparation of chemotherapy which must be entrusted to licensed pharmacists, pharmacy technicians, physicians, or nurses with specific skills acquired as a result of specific education and training programs. Before preparation, a second person has the task of independently verifying the patient's identifiers, the name of the drug, the dose, the route of administration, the rate of administration, the calculation of the dose, the treatment cycle, and the cycle day.

Upon preparation, one of the two operators must verify the drug vial(s), the concentration, the drug volume or weight, the diluent type and volume, and the administration fluid type, volume, and tubing.

Before each chemotherapy administration, at least two practitioners have to verify eight essential elements: the drug name, the drug dose, the infusion volume, the rate of administration, the route of administration, the expiration dates and/ or times, the physical integrity of the drugs, the rate set on the infusion pump.

Chemotherapy drugs — and ideally any drug used to treat cancer patients-must be labeled immediately upon preparation, including the following details [10]: patient's name, a second patient identifier, full generic drug name, drug dose, drug administration route, total volume required to administer the drug, date the medication is to be administered, expiration dates and/or times, sequencing of drug administration and total number of products to be given when medication is provided in fractionated doses (e.g., one of five, two of two), and a warning about storage and handling.

Before the administration, the practitioner administering the treatment has to confirm it with the patient, reporting at least the following information: drug name, infusion time, route of administration, and any infusion-related symptoms they should report. At least two individuals, in the presence of the patient, must verify the patient identification using at least two identifiers. Documentation of chemotherapy administration must report the verification of the aforementioned eight elements that had to be checked before the administration (see above). Procedures to manage extravasation must be defined and must follow the most up-to-date guidelines. The antidote sets must be accessible within the appropriate timeframe. After the administration of the treatment, appropriate procedures must be adopted to monitor adherence, toxicity, and possible complications.

Some studies have analyzed the different clinical and management settings in which the medication errors were reported. Interestingly, most them were intercepted and corrected before they reached the patient [11-14]. Pharmacists or 
nurses usually detected order-writing errors and informed clinicians, thus preventing harm to the patient. Therefore, no harm occurred as a result of error, in part thanks to the diligence of the staff and the special alertness of the team in questioning any unclear or ambiguous orders. This proactive approach allows the team to frequently intercept errors before they cause serious AEs. In addition, it produces cultural changes among health professionals, consequently improving medication prescription processes (e.g., development and adoption of specific computerized and noncomputerized order templates). In contrast, behaviors such as the hiding of errors or the sanctioning attitudes are an obstacle to the creation of a culture of patient safety among health professionals. Of note, hospital-based or center-based incident reporting systems have often performed better than nationwide systems, in which the problem of underreporting is more common $[15,16]$.

\subsection{Safety Practices and Implementation Strategy in Clinical Oncology}

Different strategies have been proposed in order to reduce the risk of medication errors during cancer care or to mitigate their effects or harm to the patient (Table 18.1). Therapeutic orders should be drawn up from standardized and continuously monitored dictionaries. By using computer technology, errors stemming from a misunderstanding of handwriting can be avoided. In the literature, there are some recommendations that mainly concern the way in which to report orders related to the prescription of chemotherapy. In particular, some information should always be present in each order report, including patient data such as name, height, weight, and body surface area, and treatment characteristics such as route of administration, timing, and dose. Furthermore, specific checklists that include

Table 18.1 Strategic measures to improve safe management of anticancer medication ${ }^{\mathrm{a}}$

\begin{tabular}{|c|c|}
\hline Strategic areas & Examples of intervention measures \\
\hline Training & $\begin{array}{l}\text { - All health professionals involved in treating cancer patients (i.e., physicians, pharmacists, } \\
\text { nurses, technicians) must have the necessary knowledge and skillset to perform their } \\
\text { functions. } \\
\text { - Continuous education programs must be aimed at all staff members. } \\
\text { - Periodic audits must be implemented for accreditation by authorized independent } \\
\text { organizations. }\end{array}$ \\
\hline Resources & $\begin{array}{l}\text { - Staff and technological resources must be commensurate with the volume of work. } \\
\text { - High pressure placed upon care staff must be avoided. } \\
\text { - Information and communication technology-based integrated system focused on the } \\
\text { management of cancer patients has to be part of the hospital infrastructures. }\end{array}$ \\
\hline $\begin{array}{l}\text { Operating } \\
\text { procedures }\end{array}$ & $\begin{array}{l}\text { The healthcare setting must have in place standard operating procedures which include } \\
\text { strategies for preventing errors. } \\
\text { The operating procedures must contain clear definitions of the processes for prescribing, } \\
\text { pharmaceutical reviewing, preparing, dispensing, administering, and monitoring anticancer } \\
\text { therapy. } \\
\text { - Responsibilities of each member of the staff must be clearly indicated in each process. } \\
\text { The Center Cancer must have up-to-date dictionaries with evidence-based treatment } \\
\text { protocols, clearly and unambiguously written, and accessible to all health professionals } \\
\text { involved in cancer patient care. }\end{array}$ \\
\hline $\begin{array}{l}\text { Informing the } \\
\text { patient and } \\
\text { caregivers }\end{array}$ & $\begin{array}{l}\text { The involvement of the patient and one or more caregivers is encouraged in order to facilitate the } \\
\text { care process. To this end, it is crucial to pay attention to the completeness and clarity of the } \\
\text { information provided. }\end{array}$ \\
\hline $\begin{array}{l}\text { Process of } \\
\text { prescribing the } \\
\text { treatment }\end{array}$ & $\begin{array}{l}\text { - Standardization of treatment orders. } \\
\text { The use of a computerized physician order entry (CPOE) system integrated into the medical } \\
\text { records, equipped with a clinical decision support system (CDSS) that allows to minimize } \\
\text { prescribing errors (e.g., dose calculation alerts, adjustments according to clinical situations, } \\
\text { allergies, maximum doses). }\end{array}$ \\
\hline
\end{tabular}


Table 18.1 (continued)

\begin{tabular}{|c|c|}
\hline Strategic areas & Examples of intervention measures \\
\hline $\begin{array}{l}\text { Process of } \\
\text { preparing/ } \\
\text { dispensing the } \\
\text { treatment }\end{array}$ & $\begin{array}{l}\text { - The staff of the pharmacy must follow standard guidelines or protocols related to the } \\
\text { composition, reconstitution, dilution, stability, labeling of each drug used at the site. } \\
\text { - Anticancer therapy must be prepared by the pharmacy staff in a safety cabinet, which can be } \\
\text { automated or not, for one patient at a time, and each drug must be prepared individually. } \\
\text { - Preparation of anticancer therapy should be completed by the pharmacy staff in such a way } \\
\text { that no further preparation is required by the health professional responsible for administering } \\
\text { the treatment (i.e., nurses). } \\
\text { - A standardized labeling method must be used for ensuring easy identification of patient, } \\
\text { medication, route, and dose. Labels must be printed (not handwritten). } \\
\text { The components of intravenous mixtures of cancer drugs must be verified using bar codes or a } \\
\text { similar system. The preparation phase of cytotoxic drugs should be centrally managed within } \\
\text { the hospital facility and guaranteed by dedicated pharmacists and technicians. }\end{array}$ \\
\hline $\begin{array}{l}\text { Process of } \\
\text { administering } \\
\text { the treatment }\end{array}$ & $\begin{array}{l}\text { - Anticancer therapy must be administered by a qualified member of nursing staff. } \\
\text { - Before each drug administration, patient ID must be verified by the nurse in the presence of } \\
\text { the patient, using at least two identifiers. } \\
\text { - In addition, an expert nurse must confirm the treatment with the patient. Drug name, infusion } \\
\text { time, route of administration, and infusion-related symptoms must be reported, establishing } \\
\text { any symptoms the patient must promptly report. }\end{array}$ \\
\hline $\begin{array}{l}\text { Monitoring } \\
\text { process }\end{array}$ & $\begin{array}{l}\text { After anticancer drug administration, patients must be monitored for adherence, toxicity, and } \\
\text { complications. }\end{array}$ \\
\hline
\end{tabular}

${ }^{\mathrm{a}}$ Many of these statements are also appropriate for radiation therapy.

safety parameters to be evaluated before the prescription should always be available. Examples of such checklists are forms with information on white blood cell counts, creatinine clearance, liver function indices, and drug interactions, among others.

It should be acknowledged that computer technology does not eliminate the risk of errors. Although Computerized Physician Order Entry (CPOE) systems and Clinical Decision Support Systems (CDSS) may limit some specific errors linked to incorrect order entry, many order entry alerts can be ignored or manually bypassed by physicians.

Prescription errors can be sensibly reduced by $55-80 \%$ by a CPOE system with the aid of a CDSS [17]. The introduction of computerized information systems in clinical practice and the use of medical records have been reported to have positive effects on the reduction of clinical risk, not only in relation to the cycle of medication (i.e., planning of treatment, prescription, preparation, administration, follow-up) but also for diagnostic and therapeutic evaluation in general.

The importance of documenting the occurrence of errors is independent of the extent of the damage caused to the patient or even whether any damage has been caused.

It is well known that underreporting of errors is widespread; errors that cause less serious damage often go untracked. However, the importance of providing information on near-miss or lesser injury events is increasingly clear. Policies that start from metrics and reporting are essential for improvement and the appropriate use of data is extremely useful when implementing management practices that target-specific risks.

Barriers to compliance with reporting include low staff awareness, cumbersome interfaces for documenting and sharing data among healthcare professionals, the perception of wasted time and uselessness, or fear of repercussions in the form of punitive measures.

On the other hand, strategies for improvement include simplification, standardization, and use of information technology.

Patient safety must not be limited hierarchies. Everyone must be involved in proactive error prevention. The physician, nurse, and pharmacist should all double-check therapeutic prescriptions $[5,18]$. Patients, in turn, must be fully informed about the characteristics of the therapy and its administration. Everyone must feel encouraged 
to express any doubts. Each must share their work with the others; this is the key to success in preventing a large proportion of potential errors.

\subsection{Radiotherapy}

Radiotherapy (RT) is one of the major treatment options in cancer management and "it is widely known to be one of the safest areas of modern medicine, yet, for some, this essential treatment can bring harm, personal tragedy and even death" (Sir Liam Donaldson).

It is estimated that between one half and two thirds of new cancer cases receive RT [19], which is used with curative intent in $75 \%$ of the cases that receive it. RT is a highly effective treatment option for palliation and symptom control; however, its adverse effects are quite common.

RT has distinctive risk features owing to the invasiveness of the irradiating techniques used and to the seriousness of neoplastic disease [20]. The RT process is complex and makes use of highly specialized technical equipment. The technical advancement has played a decisive role for precision in treatment delivery, creating highly conformal dose distribution with steep dose gradients [21]. Whatever the changes might have been, the objective remains the same: to eradicate tumors and to eliminate all cells in the regions at risk with minimized normal tissue toxicity [22]. The radiation treatment process is represented can be broken down into a sequence of steps. A high level of accuracy is needed at every step for maximum tumor control with minimal risk to normal tissue, defined as Organ at Risk (OAR) [23] (Fig. 18.1).

Over the last two decades, numerous studies have reported an association between dosimetric parameters and normal tissue outcomes. In 2007, a joint task force of physicists and physicians was formed with the support of the American Society for Therapeutic Radiology and Oncology (ASTRO) and the American Association of Physicists in Medicine (AAPM) to summarize in the QUANTEC the available data in a format useful to clinicians and to update and refine the estimates provided [24, 25]. Recently, PENTEC
(Pediatric Normal Tissue Effects in the Clinic) has tried to explore and define normal tissue tolerance in developing children as a function of dose and volume of radiation, type and scheduling of chemotherapy, and surgery. This information can ideally be used to inform radiation oncologists, patients, and parents of the risks and benefits of multimodality therapy involving radiation therapy, to define radiation dose constraints for treatment planning, and to propose new research directions [26].

In general, RT-related symptoms depend on the site, volume irradiated, technique, total dose, dose fractionation, age of patient, concurrent therapy, and biology of involved tissue. RT adverse effects are classically divided into acute adverse effects (i.e., arising during the treatment and lasting for about 3 months) and late ones (i.e., arising 6 months after treatment). RTOG/ EORTC hoped to standardize the way of reporting late effects on both sides of the Atlantic [27]. This has been succeeded by the CTCAE scale (Common Toxicity Criteria for Adverse Events), whose most prominent features are the merging of early and late effects criteria into a single uniform document and the development of criteria applicable to all treatment methods (e.g., chemotherapy, RT, surgery, new biotechnological drugs) [28]. RT-related symptoms can be divided into general symptoms associated with the procedure or disease and specific symptoms related to the site of irradiation. Among the former, patients often indicate fatigue as the most disturbing adverse effect of radiotherapy, ahead of pain, nausea, and vomit. Fatigue correlated with radiotherapy occurs acutely in $80 \%$ of patients and chronically in $30 \%$ of patients. For this reason, patients should be evaluated for this symptom at regular intervals. The prevalence of depression among cancer patients is extremely variable, ranging from $0 \%$ to $60 \%$ in the different case studies, depending on study criteria, methodology, and populations. Depression is associated particularly with cancers of the oropharynx, lung, breast, brain, and pancreas, and rarely with gynecologic tumors and colorectal cancers. Since comorbidity and treatment regimens are usually combined with chemotherapy, 


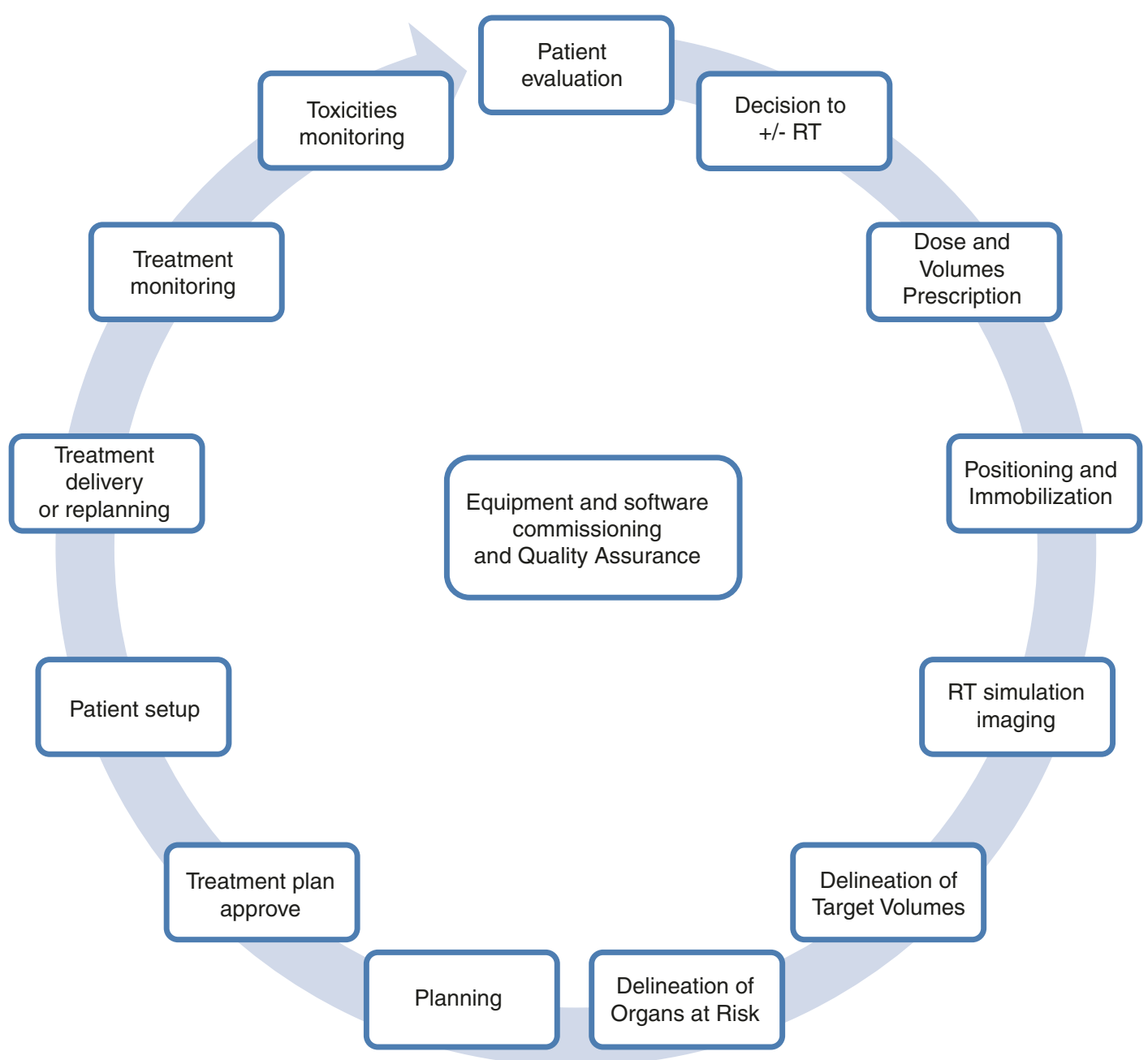

Fig. 18.1 Radiotherapy process of care from the first evaluation to follow-up. (Modified from WHO World Alliance for Patient Safety Radiotherapy Safety Expert Consensus Group)

it is often difficult to evaluate the direct effect of radiotherapy on depression as well as several other symptoms [29].

Specific symptoms relating to the irradiation of specific regions involve several organs. Here, we summarize only the main ones.

Acute and late effects of RT on the central nervous system are common and represent a significant source of morbidity. In particular, patients with tumor-related neurocognitive dysfunction may exhibit exacerbated deficits after RT [30].

Dermatitis caused by radiation is a common adverse effect of radiotherapy, often complicating treatment of breast, prostate, perineum, and head and neck cancers. It is, however, difficult to evaluate the real burden of the phenomenon as clinical practice in this field is biased by unreliable and contradictory evidence [31]. Early reactions include skin rash, and dry and humid exfoliation, while delayed events include pigmentation changes, telangiectasias, hair loss, atrophy, and ulcerations.

With regard to vascular diseases due to radiotherapy, patients who received left-sided radiotherapy as compared with those receiving right-sided radiotherapy experienced increased risks of developing coronary heart disease (RR $1,29)$ and cardiac death (RR 1,22). Radiotherapy 
for breast cancer was associated with cardiac absolute risk increase of 76.4 cases of coronary heart disease and 125.5 cases of cardiac death per 100,000 person per year [32].

With regard to effects on the lungs, pneumonia due to radiation occurs in $5.15 \%$ of patients undergoing radiation for mediastinal cancers as well as breast and lung cancers. The risk is directly associated with the volume of the irradiated lung, intensity of radiations, fractionation and concurrent chemotherapy [30].

Toxicity of the digestive tract deriving from damage to the salivary glands is common in the radiation of the head-neck area, especially in the case of concurrent chemotherapy [33]. Esophagitis is also a common, early adverse effect whose frequency grows with increasing radioactive doses and in the presence of concurrent chemotherapy [34]. Acute enteritis after radiotherapy is usually a self-limiting process, mitigated by correcting the diet and administering anti-diarrheal medications. The main risk factors include the completion of chemotherapy before the radiation of the upper abdomen and the width of the irradiated areas. Patients at higher risk are those who receive total-body radiations [35].

Acute bladder infection, including the most severe hemorrhagic cystitis, is a less common effect of radiotherapy, and the risk is elevated by concurrent chemotherapy [36].

Sexual dysfunctions, including impotence, are common after radiotherapy for cancers of the prostate and, to a lesser extent, the colon-rectum. It mainly concerns older patients, who exhibit a higher frequency of prostate cancer diagnosis. Erection dysfunction is more common with brachytherapy and with external radiotherapy [37]. In women, sexual dysfunctions are more common after radiotherapy for cervical and endometrial cancers. The adverse effects include a lower sex drive, vaginal dryness, and an overall sexual dissatisfaction [38].

Of no less importance is the role of RT in the multimodal treatment for many childhood tumors, offering an important opportunity when the limits of surgical possibilities have been reached. Due to growing tissue, children are particularly sensitive to radiation-induced adverse effects and the induction of secondary malignancies [39]. The significant developments in radiation therapy techniques together with risk-adapted treatment strategies have proven to offer advantages for the treatment of children in limiting dose exposure. The basic principle of pediatric radiotherapy is to tailor treatment intensity according to the individual risk profile (Fig. 18.2).

Quality Assurance (QA) programs improve the effectiveness of RT programs. Indeed, QA in RT involves all the procedures that ensure consistency of the dose/volume prescription, together with minimal dose to normal tissue and minimal exposure of the health operators [40].

Clinical, biological, and technical characteristics, dose delivery, organizational and training aspects can all have an impact on the efficacy, safety, and risk of the treatment [41].

The reference model in literature for clinical risk management is "clinical governance." It indicates all the activities of healthcare compa-
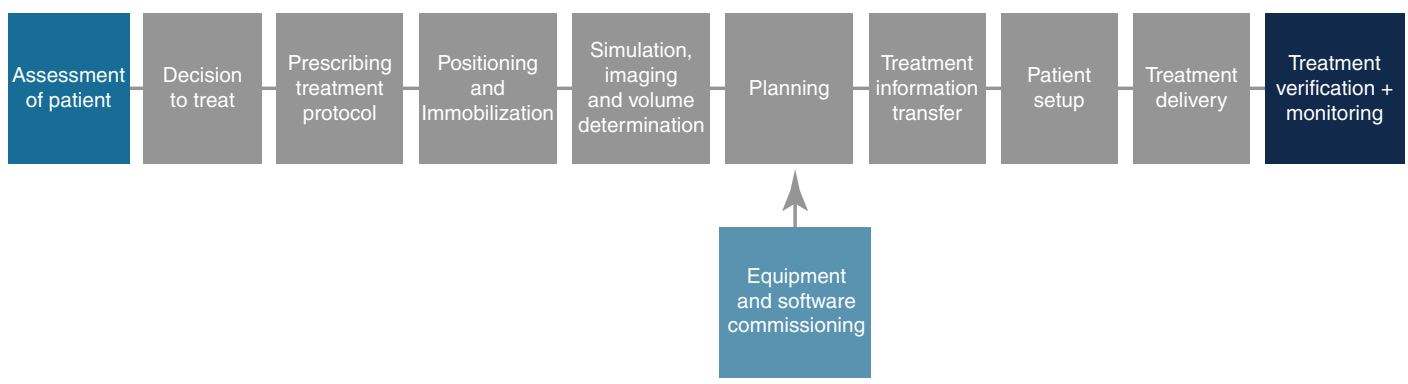

Fig. 18.2 Risk-adapted RT in pediatric cancer patients. The process should take in account the host characteristic (children/adolescent), the tumor site and volume, the treatment planned (photons, protons, multimodality treatment, etc.), the specific risk of organ dysfunction and finally the risk of secondary malignant neoplasm (SMN) 
nies that are aimed at building relationships between different components with clinical and organizational responsibilities, based on quality, safety, and continuous improvement.

Typically, errors include unauthorized acts, operative errors, equipment failures, initiating events, accident precursors, near-misses, and other mishaps [42]. The event can occur anywhere in the entirety of the patient's clinical care process. Most of the errors are discovered in setup/treatment and during follow-up. There are still errors that are not covered by regular QA checks so individual clinics should perform a risk analysis of their own practice, classifying and learning from mistakes, to determine appropriate testing frequencies that maximize physicist time efficiency and patient treatment quality and to improve existing processes or implement new workflows [43]. The detection of errors is essential in the RT process as it minimizes the risk of repetition, activates mechanisms of correction, and instills in the staff a drive to improve the quality of daily activities.

The potential for errors in radiotherapy is high as it involves a complete patient pathway with many links in the chain. The interaction of many healthcare workers collaborating on highly technical measurements and calculations can in itself present a risk of error.

The United States Nuclear Regulatory Commission (NRC) maintains a large database of RT incidents and has estimated that about $60 \%$ or more of RT incidents are due to human error [44].

Beyond the rare cases in which there is inappropriate indication of treatment, there is still potential risk inherent to the treatment method. Three types of risk and consequent litigation exist: (1) correct RT treatment without the patient having given consent or being correctly informed of the risks and possible (potentially serious) side effects; (2) correct RT treatment not accompanied by a satisfactory therapeutic response in terms of tumor control and OAR sparing; (3) incorrect RT treatment [20].

An example of the first type is the lack of adequate explanation of fertility problems related to pelvic irradiation. Of the second type, possible, subjective biological factors or unrecognized variables can be considered; for example, the exclusive use of RT treatment instead of multimodality treatment is less justified in younger patients to limit and/or avoid serious, late side effects. The third case-incorrect RT treatmentfalls under malpractice due to inexperience, carelessness, or negligence. Such malpractice leads to civil and criminal liability where demonstrated and where there is definite cause of injury to the patient.

The WHO presents in "Radiotherapy Risk" a summary of all widely reported and major radiotherapy incidents that led to significant adverse events for patients (such as radiation injury and death) and which have occurred in the last three decades (1976-2007) [41]. In total, 3125 patients were affected, of whom 38 patients $(1.2 \%)$ were reported to have died due to radiation overdose toxicity. Overall, 55\% of incidents occurred in the planning stage, while $45 \%$ occurred during the introduction of new systems and/or equipment such as megavoltage machines.

The ROSIS database, a voluntary safety reporting system for Radiation Oncology for RT incidents in Europe [45] reports a total of 1074 incidents between 2003 and 2008: 97.7\% were related to external beam radiation treatment delivered with Linear Accelerator or Cobalt, and $50 \%$ resulted in incorrect irradiation. Many incidents arise during the pretreatment phase but only about $25 \%$ of the reported process-related incidents were detected prior to treatment. Of the cases in which an incident was not detected prior to treatment, an average of $22 \%$ of the prescribed treatment fractions were delivered incorrectly. The most commonly reported detection methods were "found at time of patient treatment" and during "chart-check." The majority of the reported incidents $(56 \%)$ were detected by radiation therapists in the treatment unit. While the majority of incidents that are reported are of minor dosimetric consequence, on average they affect more than $20 \%$ of the patient's treatment fractions.

Criteria for assessing treatment correctness are the following: (1) correspondence of the treatment schedule for administration of RT dose and techni- 
cal performance by the radiographer or portal images or CT on board (IGRT); (2) identification of the most restricted field of radiation possible, taking into account the assistance provided by modern diagnostic imaging devices in preparing the treatment plan and centering; (3) safety through quality control of the device and exactness of the dose delivered, which mainly falls under the competence of the medical physicist.

The definition of organized protocols is fundamental; they should provide methods for the constant monitoring of radiotherapy devices and identification of the person responsible for each process. To prevent any risk of incorrect calibration, devices should be submitted to quality control at the beginning of each new working cycle. Specific competence of the radiotherapist for the organ or system being treated has become accepted among the current criteria for treatment quality.

Due to rapidly increasing complexity during the last few years, comprehensive QA has become even more important for treatment planning software, information handling, and treatment delivery.

The main health professionals involved in the delivery of RT are Radiotherapist Physicians, Radiation Therapists, and Medical Physicists, while the final guarantor of the process is the Radiotherapist Physician, who must acquire the patient's informed consent, which is a process, not a form. The need to obtain informed consent for treatment is based on the patient's right to self-determination and the fiduciary relationship between the patient and physician [46]. The Radiotherapist assumes responsibility for protecting the patient from errors and damage caused by the incorrect use of ionizing radiation (whether or not associated with drugs).

In the present era, RT is being enriched by big digital data and intensive technology. Modern radiotherapy departments transfer a great quantity of data from diagnosis to planning and treatment machines. The new technologies can help in reducing the risks but, when not used correctly or if the personnel is not adequately trained, can paradoxically act as a new source of error for manual procedures [47].
Multimodality image registration, intelligenceguided planning, real-time tracking, imageguided RT (IGRT), radiomics, and automatic follow-up surveys are the products of the digital era. Enormous amounts of digital data are created in the process of treatment, carrying both benefits and risks. Generally, decision-making in RT tries to balance these two aspects based on the archival and retrieving of data from various platforms.

Modern risk-based analyses show that many errors occurring in radiation oncology are due to failures in workflow $[43,48]$. Medical imaging is crucial to RT; its application, referred to as IGRT, encompasses tumor diagnosis, staging, prognosis, treatment planning, radiation targeting, and follow-up care [49]. Setup errors, ranges of organ motion, and changes in tumor position and volume are most likely to be detected during an RT course with frequent imaging, which is becoming an essential requirement in order to attain the best local control of tumors and OAR sparing [50]. Various studies indicate that inconsistencies in anatomy contouring may be larger than errors in the other steps of the treatment planning and delivery process [51]. Some semiautomatic and automatic contouring methods have been proposed, such as probabilistic atlases and machinelearning technologies, to minimize manual input and increase consistency in delineating clinical target volume (CTV) and planning target volume (PTV). Image guidance is suggested, allowing margin reduction to several millimeters and dose escalation while maintaining the sparing of the OAR. However, respiratory motion and artifacts may distort target volume. This can be compensated for with the use of four-dimensional CT (4DCT) or instruments like "breath holding" that are able to facilitate the delivery of RT to a dynamic target rather than to a static volume. Tumor motion management has been a part of the IGRT effort and it markedly impacts radiation dose and volume of irradiated, normal tissue, as well as secondary cancer risk (SMN) [43].

The risks for RT-related SMN depend on the technique used (e.g., the risk for all tumors increases with the increase of number of fields, with higher energy, and with modulated/dynamic techniques) and vary in different organs and 
tissues. Both size and shape of the PTV influence the risk of secondary malignancy. If we consider only SMN causing risk for life (e.g., sarcoma, carcinoma), they usually occur in healthy tissues near volumes irradiated with high-intermediate doses [52-55]. Of 30,000 adult patients treated between 1969 and 1989 with RT, 203 were readmitted for RT due to an SMN. About $70 \%$ of SMNs occur in intermediate-high dose regions. The stomach, lungs, and colon are the most common sites for SMN after radiation exposure [54].

With the use of modern RT photon techniques such as intensity-modulated radiation therapy IMRT, the above-target, high dose volumes in healthy tissue have been drastically reduced compared with conventional 2D- or 3D-RT. One might expect a decrease in the number of sarcomas induced and (maybe with less certainty) a small decrease in the number of carcinomas. By contrast, the move from 3D-RT to IMRT involves more fields, and the dose-volume histograms show that as a consequence a larger volume of normal tissue is exposed to lower doses [56] .

The greatest challenge in determining risk is that secondary cancers after RT have onset latencies of 5-10 years for leukemia and about 10-60 years for solid tumors after the initial treatment. Only longer term follow-up will allow a true assessment of the SMN risk. A risk-adapted strategy can be taken to optimize the routine follow-up, the screening frequency, and the followup duration.

While IMRT marks the crowning achievement of photon-based external beam radiation therapy, the next step in improving physical-dose distribution naturally points to heavy-charged particle beams using the clinical application of Bragg peak. The recent introduction of particle therapy into clinical practice offers to minimize the radiation dose to healthy tissues near cancer targets, offering significantly lower second cancer incidence rates than photon-based therapy [53].

Problems related to the harmonious development of RT in a growing multidisciplinary context lie at the center of a growing interest to scientific groups. The scientific community of radiation oncologists is generally used to monitor the acute and late side effects related to treat- ment, and consequently to adopt and to adapt methods from clinical Risk Management. The optimal use of preventative and integrated treatment interventions based on a logical and systematic methodology is carried out through multiple, linked phases to improve the quality of health services and guarantee patient safety. Risk management is effective through the identification, knowledge and analysis of risks and errors (via report systems, folder review, use of indicators, etc.), identification and correction of causes (via Root Causes Analysis, Process Analysis, Failure Mode and Effect Analysis, etc.), monitoring of preventive measures, and implementation and active support of proposed solutions.

Only integrated risk management can lead to changes in clinical practice and promote the growth of a healthcare culture that is more attentive to patients and operators. It contributes indirectly to the decrease in the cost of services and encourages the allocation of resources towards interventions aimed at developing safe and efficient health organizations and structures.

\subsection{Safety Practices and Implementation Strategy for Radiotherapy}

Modern radiotherapy depends considerably on the transfer of patient data between different operative units, information systems, and staff of different disciplines.

The steps of the process are presented as follows in "Safety is No Accident-ASTRO" (American Society for Radiation Oncology, 2019):

- Assessment of patient, or patient evaluation

- Radiation treatment preparation

- Clinical treatment planning

- Therapeutic simulation

- Dosimetric treatment planning

- Pretreatment quality assurance and plan verification

- Radiation treatment delivery

- Radiation treatment management

- Follow-up evaluation and care 
Care has to be taken at each step and general strategies are also needed to reach the theoretical goal of no accidents occurring.

One basic strategy for the improvement of safety in the radiotherapy process is the development of a stable incident reporting and analysis structure. This could be designed in two steps. First, a systematic cross-check of the procedures should be established, at least at the end of the main subprocesses: clinical assessment and decision, target volume and organ at risk outlining, dosimetric treatment planning, and pretreatment checks. As a second step, it is important to instill in all operators the idea of error discovery and disclosure. This part of the process requires that all professionals (i.e., physicians, physicists, radiation therapists, etc.) are clearly informed about the potential advantages of a mandatory and tightened-up second check (i.e., a crosscheck) of their actions, in order to create or maintain a no-blame culture within the department. To improve the global effectiveness of the check, every operator should be encouraged to record potential errors and report them within a wellstructured reporting system or to dedicated professionals.

As stated by several authors, the quantitative and qualitative understanding of mistakes, gained by reporting, allows professionals to identify potential, necessary corrections to their organization or its processes, or to the technology available, in order to prevent future incidents.

If no incidents are known, that is, if operators do not report problems, no improvement will be possible.

\subsection{Volumes-Outcomes Relationship in Surgery}

Patient safety in oncology should also be considered from the perspective of the outcomes associated with complex surgical activities or the procedures that comprise a broader therapeutic approach, in order to find a significant application to solid tumors. The risk for cancer patients undergoing surgical procedures are linked, in the first place, to the generic risks that are typical of each surgical procedure, such as infection, selection of the wrong site, etc.; however, this section discusses only the risks associated with outcomes such as complications, hospitalization within 30 days from surgery, or death for surgery performed in specialized surgery centers, which differ by number of performed surgeries.

Since the earliest observations published about a century ago [57], a vast amount of studies have come to the conclusion that higher volume correlates to better outcomes in a number of medical and surgical procedures, especially in highrisk surgery. The relation between workload and the latter was regarded as a key factor in the regionalization of healthcare providers, particularly those offering surgery with a higher technology content [58]. For an overview of the medical and surgical specialties for which volumes-outcomes relationship has been found, three recent works [59-61] are available in literature. The latest report [59] highlighted for surgical procedures in oncology a positive association of considered outcomes with a high hospital volume for 14 out of 18 studied conditions (i.e., cancers of the colon, colon-rectum, esophagus, breast, ovaries, pancreas, lung, prostate, kidney, bladder, and head and neck), while for other surgical procedures no sufficient evidence was found to evaluate the association (i.e., for testis, brain, and pediatric cancers).

Workload volume thus acts as a proxy measure for various processes and provides characteristics that in turn may directly influence outcomes. The causes of this association have been investigated since the first statistical evidence of an association with mortality [62]. It has been hypothesized that for high-risk surgical procedures with relatively short lengths of stay the relationship could be largely explained by surgeon volume. A systematic review recently investigated whether high-volume surgeons of various surgical specialties perform better surgeries, (i.e., the surgeon's volume-outcome relationship) [61]. A positive volume-outcome relationship is apparent for most procedures/conditions (e.g., colorectal cancer, bariatric surgery, 
and breast cancer) and can be related to the "learning curve" of a surgeon. The simple concept of "learning by doing" and the idea that "practice makes perfect" are often used to exemplify this notion. A drawback of this work is that these results are partly based on systematic reviews with methodological weaknesses, in particular the possible risk of bias in the primary studies. On the other hand, the hospital volume-outcome relationship has been investigated, as in one recent, German work [63]. Policy makers need good evidence for policy making around the standardization of surgical procedures. For procedures requiring an extended length of stay, intensive care unit admission, and/or multidisciplinary inpatient or outpatient care (i.e., esophagectomy, pneumonectomy, hepatobiliary resection), the relationship can be largely explained by hospital volume, due to a large multidisciplinary team and many hospital processes of care required to achieve high-quality care.

Drawing parallels between the "experience curve" describing a decline in unit costs as function of cumulative production experience, a relation well documented in industrial economics, the Author underlines that a substantial number of deaths could be averted if all patients were treated in hospital having results similar to those of high-volume hospitals. Such consideration would represent a strong support in favor of regionalizing certain surgical procedures. To make a long story short: larger volumes lead to better outcomes. At a more fundamental level, one may ask whether a model focusing on volumes may be appropriate. Many questions arise concerning the relationship between outcomes and experience accumulated over several years, the significance of the procedure alone compared with the more general set of procedures to which a specific procedure belongs to. Moreover, it should be clarified whether to regionalize operations into larger medical centers even when smaller hospitals with high volumes of specific procedures exists. This perspective raises some issues with medical students and young physicians learning curve. In summary, volume alone probably does not automatically result in better performance, but acts as a proxy measure for various processes and provides characteristics that in turn may directly influence outcomes.

In a large national dataset of Medicare patients, it was found that, after more than three decades, both absolute and relative differences in adjusted mortality persist between hospitals with the lowest and highest volume [64]. Two recent overviews of systematic reviews [61, 63] found evidence synthesis based on systematic reviews instead of primary studies. This result supports a positive volume-outcome association for the surgeon/practitioner performing most procedures/ conditions. However, results are partly based on systematic reviews with methodological weaknesses, as to say the lack of consideration of the risk of bias in the primary studies [61]. The authors suggest that forthcoming reviews, to compare better findings across studies, should pay more attention to methodology specific to volume-outcome relationship.

A recent systematic review and meta-analysis on volume-outcome associations in head and neck oncology identified six studies that assessed long-term survival with conflicting results of limited external validity [65]. Of the studies analyzed, only one was able to assess both hospital and surgeon volume concurrently. This is a significant limitation because it has been previously demonstrated that, depending on the procedure, either surgeon or hospital volume can explain most of the effect on outcome. Thus, these studies cannot delineate between the relative importance of hospital- and surgeon-volume effects [65].

Considering head and neck cancer patients, the same authors took into account data coming from a single payer national healthcare system. The results of the meta-analysis were consistent with the hypothesis: for head and neck cancer resections that often require an extended hospital stay and inpatient and outpatient multidisciplinary care delivered by a large team, the relationship appears to be explained not only by surgeon volume but more strongly by hospital volume [66]. 


\subsection{Case History}

1. A 55-year-old woman with a recent diagnosis of early-stage triple negative breast cancer was admitted to the breast unit of a National Cancer Center to receive the second cycle of a regimen with epirubicin and cyclophosphamide. Although an apparently correct order was written by the physician, and the pharmacist entered the right dose of both drugs through the computerized provider order entry system, 7 days after the treatment administration the patient experienced a severe toxicity (i.e., febrile neutropenia and G3 stomatitis). Therefore, a deep verification process was started in order to exclude potential medication errors responsible for the observed side effects. In particular, a rigorous examination of the medical record together with the analysis of the various steps of the therapeutic course were performed. Notably, the second pharmacist, who was charged with independently verify the computerized order, signed the drug preparation as correct. In turn, before treatment administration, two nurses were charged to appropriately check for the pharmacists' signatures, and to independently compare the dose on the medication to the written physician order. Both nurses confirmed the correctness of the process, and the drug administration was initiated. Subsequently, before all of the medication had infused and according on standard procedures, the dose on the medication bag was compared to the written order, but no errors were found. Appropriately, every step about treatment prescription, preparation, and administration were adequately reported on the medical chart. This approach allowed us to recapitulate the whole event and to identify the error that had consisted into a wrong programming of the interval between the two cycles of therapy, administered at a distance of 2 weeks instead of 3 . Therefore, the patient received an unintentional dose-dense regimen without support of pegfilgrastim. The physician was misled by the normal values of the blood count before starting the second cycle of chemotherapy. This is a clear example of an error due to a failure of a CPOE system to provide a proper alert about the wrong interval between two cycles of treatment, and it underlines the importance of a correct set of protocols with each distinct variable unequivocally detailed.

2. Patient undergoing two subsequent treatments with tomotherapy on two different areas of the same anatomical site. For the two treatments, two different immobilization and positioning masks were made with different positioning points. The IGRT (Image-Guided Radiation Therapy) procedure, mandatory before each treatment, requires the acquisition of images with the patient wearing the mask, positioned on the treatment bed, and a comparison of the acquired images with the simulation images, on which the dosimetry plan was conducted. For this patient, the IGRT procedure indicated an anomaly of a few centimeters along the cranial-caudal direction and a different rotation of the patient body on the transversal plan. The repositioning needed to return to the correct anatomical reference point was interpreted as a positioning error of the LASER during the planning phase. In addition, no investigation was conducted on the different rotation of the patient's body on the transversal plan. The repositioning according to the IGRT procedure movements was conducted and the patient received therapy in three sessions. After the third session, an evaluation of the images revealed that the positioning preIGRT did not coincide with that of the simulation and that the repositioning post-IGRT did not guarantee the precision required in these cases. The treatment was suspended, and an investigation indicated that the immobilization and positioning mask of the first treatment had not been destroyed as by requirement, and that at the first three sessions of the second treatment, the patient had been immobilized and positioned using the same mask of the first treatment. The second treatment was modified, in consideration of what had 
happened during the first three sessions, and the patient continued therapy with the new treatment and the correct mask for the subsequent sessions without adverse effects.

\subsection{Final Recommendations}

Implementation of best practices in clinical oncology, to reduce the risk of error and preventing harm to the patient, must be seen as a priority among professionals and health organizations. The growing complexities of modern medicine require continuous updates and adjustments to meet new necessities. The safest possible administration of chemotherapy drugs requires a continuous surveillance of the methods through which the drugs are administered. The implementation of an orderly method of administration of the drugs is, today, a mandatory process of revision, if we want to obtain a significant reduction in the risk of error that can result in harm to the patient. The preparation of cytotoxic drugs can often lead to error. Therefore, it should be centrally managed in the hospitals and possibly guaranteed by dedicated technicians and pharmacists. The procedures not only must be kept in writing and shared with the team, but they also need to be monitored and revised in order to avoid the introduction of new types of errors. They can take advantage of integrated electronic systems, such as Computerized Provider Order Entry (CPOE) and Clinical Decision Support System (CDSS). Each hospital where oncology practices are provided must have a pharmacy staff trained to guarantee the appropriate preparation of drugs, which can also benefit from new technologies that include always improving monitoring systems and bar coding. The organization must enforce a safety culture among the hospital staff members, so that they can feel competent and involved in the processes of improving quality of services and preventing pharmacological errors. Patients should be encouraged to contribute to their own care through patient education programs with focus on self-evaluation and on monitoring the effect of their treat- ments. Efforts must be made with the aim of improving systems to report and analyze errors. Coherently, strong strategies must be introduced to promote inter-professional and patient-provider communication.

Concerning radiotherapy, it is necessary: to document in detail the different phases of the process; to be able to rely on adequate information systems; to work in team; to support and implement a culture of safety training; to increment a system of error reporting and near-misses; to start methods of error analysis; to support continuous education and training, especially in case of introduction of new technologies.

In conclusion, there should be an open communication among the different professionals involved in management of treatment with the aim to take inspiration from the best practices, which must be defined on the basis of scientific evidence. Moreover, the Hospital Organization must ensure a peaceful environment and a serene atmosphere, warranting necessary organizational conditions.

\section{References}

1. Rees G. Risk management in clinical oncology. In: clinical risk management, enhancing patient safety, second edition, edited by charles vincent. BMJ. 2001;197-217.

2. Pearce A, Haas M, Viney R, Pearson SA, Haywood $\mathrm{P}$, et al. Incidence and severity of self-reported chemotherapy side effects in routine care: a prospective cohort study. PLoS One. 2017;12(10):e0184360.

3. Chopra D, Rehan HS, Sharma V, Mishra R. Chemotherapy-induced adverse drug reactions in oncology patients: a prospective observational survey. Indian J Med Paediatr Oncol. 2016;37(1):42-6.

4. Miranda V, Fede A, Nobuo M, et al. Adverse drug reactions and drug interactions as causes of hospital admission in oncology. J Pain Symptom Manag. 2011;42(3):342-53. https://doi.org/10.1016/j. jpainsymman.2010.11.014.

5. Weingart SN, Zhang L, Sweeney M, Hassett M. Chemotherapy medication errors. Lancet Oncol. 2018;19(4):e191-9. https://doi.org/10.1016/ S1470-2045(18)30094-9.

6. Sledge GW. Patients and physicians in the era of modern cancer care. JAMA. 2019;321(9):829-30.

7. Knoop T, Wujcik D, Wujcik K. Emerging models of interprofessional collaboration in cancer care. Semin Oncol Nurs. 2017;33(4):459-63. 
8. Lipitz-Snyderman A, Pfister D, Classen D, Atoria CL, Killen A, Epstein AS, et al. Preventable and mitigable adverse events in cancer care: measuring risk and harm across the continuum. Cancer. 2017;123(23):4728-36.

9. Neuss MN, Gilmore TR, Belderson KM, et al. Updated American Society of Clinical Oncology/ Oncology Nursing Society chemotherapy administration safety standards, including standards for pediatric oncology. J Oncol Pract. 2016;12(12):1262-71. Erratum in: J Oncol Pract. 2017;13(2):144.

10. Trudeau M, Green E, Cosby R, et al. Key components of intravenous chemotherapy labeling: a systematic review and practice guideline. J Oncol Pharm Pract. 2011;17:409-24.

11. Serrano-Fabiá A, Albert-Marí A, Almenar-Cubells D, Jiménez-Torres NV. Multidisciplinary system for detecting medication errors in antineoplastic chemotherapy. J Oncol Pharm Pract. 2010;16:105-12.

12. Díaz-Carrasco MS, Pareja A, Yachachi A, Cortés F, Espuny A. Prescription errors in chemotherapy. Farm Hosp. 2007;31:161-4.

13. Markert A, Thierry V, Kleber M, Behrens M, Engelhardt M. Chemotherapy safety and severe adverse events in cancer patients: strategies to efficiently avoid chemotherapy errors in in- and outpatient treatment. Int J Cancer. 2009;124:722-8.

14. Ford CD, Killebrew J, Fugitt P, Jacobsen J, Prystas EM. Study of medication errors on a community hospital oncology ward. J Oncol Pract. 2006;2:149-54.

15. Fyhr A, Akselsson R. Characteristics of medication errors with parenteral cytotoxic drugs. Eur J Cancer Care. 2012;21:606-13.

16. Rinke ML, Shore AD, Morlock L, Hicks RW, Miller MR. Characteristics of pediatric chemotherapy medication errors in a national error reporting database. Cancer. 2007;110:186-95.

17. Nerich V, Limat S, Demarchi M, et al. Computerized physician order entry of injectable antineoplastic drugs: an epidemiologic study of prescribing medication errors. Int J Med Inform. 2010;79:699-706.

18. Schulmeister L. Preventing chemotherapy errors. Oncologist. 2006;11(5):463-8.

19. Delaney G, et al. The role of radiotherapy in cancer treatment: estimating optimal utilization from a review of evidence-based clinical guidelines. Cancer. 2005; 104:1129-37.

20. Luca A, Fileni A. Risk management in radiotherapy: analysis of insurance claims. Radiol Med. 2006;111:733-40.

21. Sullivan R, Peppercorn J, Sikora K, et al. Delivering affordable cancer care in high-income countries. Lancet Oncol. 2011;12(10):933-80.

22. Wu X. The technical infrastructure of a Modern Radiation Oncology Department. In: Radiation oncology an evidence-based approach. Berlin: Springer; 2008. p. 641.

23. Walker SJ. The management of treatment incidents: an analysis of incidents in radiotherapy. In: Faulkner K,
Harrison RM, editors. Radiation incidents. London: British Institute of Radiology; 1996. p. 29-35.

24. Emami B, Lyman J, Brown A, Coia L, Goitein M, Munzenrider JE, et al. Tolerance of normal tissue to therapeutic irradiation. Int J Radiat Oncol Biol Phys. 1991;21(1):109-22.

25. Marks LB, Ten Haken RK, Martel MK. Guest editor's introduction to QUANTEC: a users guide. Int J Radiat Oncol Biol Phys. 2010;76(3):S1-2.

26. Constine LS, Ronckers CM, Hua $\mathrm{CH}$, Olch A, Kremer LCM, Jackson A, Bentzen SM. Pediatric normal tissue effects in the clinic (PENTEC): an international collaboration to analyse normal tissue radiation dose-volume response relationships for paediatric cancer patients. Clin Oncol (R Coll Radiol). 2019;31(3):199-207.

27. Anonymous. Late effects consensus conference. Radiother Oncol. 1995;35(1):5-7.

28. https://ctep.cancer.gov/protocolDevelopment/electronic_applications/ctc.htm. Accessed 28 Feb 2020.

29. Berkey FJ. Managing the adverse effects of radiation therapy. Am Fam Physician. 2010;82(4):381-8, 394.

30. Lawrence YR, Li XA, el Naqa I, Hahn CA, Marks LB, Merchant TE, et al. Radiation dose-volume effects in the brain. Int J Radiat Oncol Biol Phys. 2010;76(3):S20-7.

31. Lam E, Yee C, Wong G, Popovic M, Drost L, Pon $\mathrm{K}$, et al. A systematic review and meta-analysis of clinician-reported versus patient-reported outcomes of radiation dermatitis. Breast. 2020;50:125-34.

32. Cheng YJ, Nie XY, Ji CC, et al. Long-term cardiovascular risk after radiotherapy in women with breast cancer. J Am Heart Assoc. 2017;6(5):e005633. https:// doi.org/10.1161/JAHA.117.005633.

33. Deasy JO, Moiseenko V, Marks L, Chao KS, Nam J, Eisbruch A. Radiotherapy dose-volume effects on salivary gland function. Int J Radiat Oncol Biol Phys. 2010;76(3):S58-63.

34. Werner-Wasik M, Yorke E, Deasy J, Nam J, Marks LB. Radiation dose-volume effects in the esophagus. Int J Radiat Oncol Biol Phys. 2010;76(3):S86-93.

35. Kavanagh BD, et al. Radiation dose-volume effects in the stomach and small bowel. Int J Radiat Oncol Biol Phys. 2010;76(3):S101-7.

36. Viswanathan AN, Yorke ED, Marks LB, Eifel PJ, Shipley WU. Radiation dose-volume effects of the urinary bladder. Int J Radiat Oncol Biol Phys. 2010;76(3):S116-22.

37. Roach M 3rd, Nam J, Gagliardi G, El Naqa I, Deasy JO, Marks LB. Radiation dose-volume effects and the penile bulb. Int $\mathrm{J}$ Radiat Oncol Biol Phys. 2010;76(3):S130-4.

38. White ID. Sexual difficulties after pelvic radiotherapy: improving clinical management. Clin Oncol (R Coll Radiol). 2015;27(11):647-55.

39. Steinmeier T, Schulze Schleithoff S, Timmermann B. Evolving radiotherapy techniques in paediatric oncology. Clin Oncol (R Coll Radiol). 2019;31(3):14250. https://doi.org/10.1016/j.clon.2018.12.005. 
40. World Health Organization (WHO). Quality assurance in radiotherapy. Geneva: WHO; 1988.

41. Radiotherapy risk profile. Technical manual. WHO; 2008.

42. Portaluri M, Fucilli FI, Gianicolo EA, et al. Collection and evaluation of incidents in a radiotherapy department: a reactive risk analysis. Strahlenther Onkol. 2010;186(12):693-9.

43. Jin F, Luo HL, Zhou J, He YN, Liu XF, Zhong MS, et al. Cancer risk assessment in modern radiotherapy workflow with medical big data. Cancer Manag Res. 2018;10:1665-75.

44. Duffey RB, Saull JW. Know the risk: learning from errors and accidents: safety and risk in today's technology. Butterworth-Heinemann Publications: Boston, MA; 2003.

45. Cunningham J, Coffey M, Knöös T, Holmberg O. Radiation oncology safety information system (ROSIS) - profiles of participants and the first 1074 incident reports. Radiother Oncol. 2010;97(3):601-7.

46. Reuter SR. An overview of informed consent for radiologists. Am J Roentgenol. 1987;148:219-22.

47. Patton G, Gaffney D, Moeller J. Facilitation of radiotherapeutic error by computerized record and verify systems. Int $\mathrm{J}$ Radiat Oncol Biol Phys. 2003;56(1):50-7.

48. Huq MS, Fraass BA, Dunscombe PB, et al. The report of Task Group 100 of the AAPM: application of risk analysis methods to radiation therapy quality management. Med Phys. 2016;43(7):4209.

49. Verellen D, De Ridder M, Linthout N, Tournel K, Soete G, Storme G. Innovations in image-guided radiotherapy. Nat Rev Cancer. 2007;7(12):949-60.

50. Dawson LA, Sharpe MB. Image-guided radiotherapy: rationale, benefits, and limitations. Lancet Oncol. 2006;7(10):848-58.

51. Segedin B, Petric P. Uncertainties in target volume delineation in radiotherapy - are they relevant and what can we do about them? Radiol Oncol. 2016;50(3):254-62.

52. Berrington de Gonzalez A, Gilbert E, Curtis R, et al. Second solid cancers after radiation therapy: a systematic review of the epidemiologic studies of the radiation dose-response relationship. Int $\mathrm{J}$ Radiat Oncol Biol Phys. 2013;86(2):224-33.

53. Hall EJ. Intensity-modulated radiation therapy, protons, and the risk of second cancers. Int $\mathrm{J}$ Radiat Oncol Biol Phys. 2006;65(1):1-7.

54. Dörr W, Herrmann T. Cancer induction by radiotherapy: dose dependence and spatial relationship to irradiated volume. J Radiol Prot. 2002;22(3A):A117-21.
55. Schneider U, Besserer J, Mack A. Hypofractionated radiotherapy has the potential for second cancer reduction. Theor Biol Med Model. 2010;7:4.

56. Hall EJ, Wuu CS. Radiation-induced second cancers: the impact of 3D-CRT and IMRT. Int J Radiat Oncol Biol Phys. 2003;56(1):83-8.

57. Codman EA. The product of a hospital. Surg Gynecol Obstet. 1914;18:491-6.

58. Luft HS, Bunker JP, Enthoven AC. Should operations be regionalized? The empirical relation between surgical volume and mortality. Surv Anesthesiol. 1980;24:395. https://doi. org/10.1097/00132586-198012000-00060.

59. Amato L, Fusco D, Acampora A, Bontempi K, Rosa $\mathrm{AC}$, Colais $\mathrm{P}$, et al. Volumi di attività ed esiti delle cure. Prove scientifiche in letteratura ed evidenze empiriche in Italia. Epidemiol Prev. 2017;41:130.

60. Halm EA, Lee C, Chassin MR. Is volume related to outcome in health care? A systematic review and methodologic critique of the literature. Ann Intern Med. 2002;137:511. https://doi. org/10.7326/0003-4819-137-6-200209170-00012.

61. Morche J, Mathes T, Pieper D. Relationship between surgeon volume and outcomes: a systematic review of systematic reviews. Syst Rev. 2016;5:204. https://doi. org/10.1186/s13643-016-0376-4.

62. Luft HS. The relation between surgical volume and mortality: an exploration of causal factors and alternative models. Med Care. 1980;18:940-59. https://doi. org/10.1097/00005650-198009000-00006.

63. Pieper D, Mathes T, Neugebauer E, Eikermann M. State of evidence on the relationship between high-volume hospitals and outcomes in surgery: a systematic review of systematic reviews. J Am Coll Surg. 2013;216:1015-25. https://doi.org/10.1016/j. jamcollsurg.2012.12.049.

64. Reames BN, Ghaferi AA, Birkmeyer JD, Dimick JB. Hospital volume and operative mortality in the modern era. Ann Surg. 2014;260:244-51. https://doi. org/10.1097/SLA.0000000000000375.

65. Eskander A, Irish J, Groome PA, Freeman J, Gullane $\mathrm{P}$, Gilbert R, et al. Volume-outcome relationships for head and neck cancer surgery in a universal health care system: universal healthcare outcomes in HNSCC. Laryngoscope. 2014;124:2081-8. https:// doi.org/10.1002/lary.24704.

66. Eskander A, Merdad M, Irish JC, Hall SF, Groome PA, Freeman JL, et al. Volume-outcome associations in head and neck cancer treatment: a systematic review and meta-analysis: volume-outcome in head and neck cancer treatment. Head Neck. 2014;36:1820-34. https://doi.org/10.1002/hed.23498. 
Open Access This chapter is licensed under the terms of the Creative Commons Attribution 4.0 International License (http://creativecommons.org/licenses/by/4.0/), which permits use, sharing, adaptation, distribution and reproduction in any medium or format, as long as you give appropriate credit to the original author(s) and the source, provide a link to the Creative Commons license and indicate if changes were made.

The images or other third party material in this chapter are included in the chapter's Creative Commons license, unless indicated otherwise in a credit line to the material. If material is not included in the chapter's Creative Commons license and your intended use is not permitted by statutory regulation or exceeds the permitted use, you will need to obtain permission directly from the copyright holder.

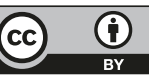

